\title{
Development and validation of a WHOQOL-BREF Taiwanese audio player-assisted interview version for the elderly who use a spoken dialect
}

\author{
Chi-Wen Chien · Jung-Der Wang • Grace Yao • \\ Ching-Fan Sheu $\cdot$ Ching-Lin Hsieh
}

Accepted: 24 June 2007 / Published online: 19 July 2007

(C) Springer Science+Business Media B.V. 2007

\begin{abstract}
A quality of life questionnaire is rarely adapted to an interview mode for people who mainly use spoken language in daily life. In Taiwan, the WHOQOL-BREF (Mandarin Chinese version) has been developed, as a self-administered questionnaire, but it cannot be applied to the majority of the elderly in Taiwan, who speak only Taiwanese (a dialect). This study adopted the audio player-assisted interview mode to develop a Taiwanese version of the WHOQOL-BREF
\end{abstract}

\section{C.-W. Chien}

Department of Occupational Therapy, School of Primary Health

Care, Faculty of Medicine, Nursing, and Health Sciences,

Monash University - Peninsula Campus, Frankston, VIC,

Australia

\section{J.-D. Wang}

Institute of Occupation Medicine and Industrial Hygiene, College of Public Health, National Taiwan University, Taipei,

Taiwan

\section{J.-D. Wang}

Department of Internal Medicine, National Taiwan University

Hospital, Taipei, Taiwan

G. Yao

Department of Psychology, National Taiwan University, Taipei, Taiwan

\section{C.-F. Sheu}

Institute of Cognitive Science, National Cheng Kung University, Tainan, Taiwan

\section{C.-L. Hsieh ( $\square)$}

School of Occupational Therapy, College of Medicine, National Taiwan University, 4F, No 17, Shiujou Rd, Taipei 100, Taiwan e-mail: clhsieh@ntu.edu.tw

\section{C.-L. Hsieh}

Department of Physical Medicine and Rehabilitation, National Taiwan University Hospital, Taipei, Taiwan specifically for Taiwanese-speaking elderly people, and followed with examinations of the reliability and validity of this version. Initially, the WHOQOL-BREF (English version) was translated into colloquial Taiwanese, and field tests confirmed the equivalence and appropriateness of the translation. A total of 228 Taiwanese-speaking elderly people were assessed using the Taiwanese interview version, of which 144 subjects were re-assessed two weeks later. Interviewers assessed each subject aided by an audio player on which all the translated WHOQOL-BREF contents were recorded. The Taiwanese interview version of the WHOQOL-BREF, except for the item related to dependence on medication, showed acceptable reliability (internal consistency, corrected item-domain correlation, and test-retest reliability) and validity (criterion-related, convergent, and discriminant validity). Confirmatory factor analyses supported the four-factor model of the Taiwanese interview version, providing evidence for construct validity. The results suggest that the Taiwanese audio player-assisted interview version of the WHOQOL-BREF was reliable and valid in assessing quality of life of elderly Taiwanese.

Keywords WHOQOL-BREF · Interview version · Quality of life

Abbreviations
WHOQOL
BREF $\quad$ World Health Organization Quality of Life

\section{Introduction}

The brief version of the World Health Organization's Quality of Life instrument (WHOQOL-BREF) has been 
widely used to assess quality of life (QOL) [1-6]. The WHOQOL-BREF was designed as a self-administration questionnaire; however, it has limited clinical application for people who are unable to read $[1,3]$. It is common to find that elderly people with elementary education or less use exclusively spoken language in their daily lives. These elderly people would benefit greatly from the development of an interview version of the WHOQOL-BREF that could assess their QOL [3].

The Mandarin Chinese version of the WHOQOL-BREF currently exists in Taiwan [7-9]. However, this tool cannot be applied to more than half of elderly Taiwanese aged over 65 years old [10], who speak only the Taiwanese dialect [11]. That is largely because these elderly Taiwanese received only a rudimentary education in the early part of the last century. In order to evaluate the QOL of this population, a Taiwanese version of the WHOQOL-BREF (WHOQOL-BREF [TV]) that is based on an oral interview mode is required.

A newly developed QOL questionnaire must be shown to be reliable and valid [12] prior to its introduction into clinical settings. This paper examined the reliability (internal consistency, corrected item-domain correlation, and test-retest reliability) and validity (criterion-related, convergent, construct, and discriminant validity) of the WHOQOL-BREF (TV) in Taiwanese-speaking elderly people.

\section{Methods}

Subjects

Subjects were recruited from 13 long-term care institutions by convenience sampling. These institutions were geographically distributed in 4 major areas of Taiwan: the northern (4 institutions), central ( 2 institutions), southern (4 institutions), and eastern ( 3 institutions) parts. Four institutions were chosen in the northern and southern areas because these regions have higher population densities, and more elderly people are thus assumed to live there. Subjects who were included met the following criteria: (1) speak only Taiwanese; (2) do not comprehend Mandarin Chinese; (3) score above 20 on the Mini-Mental State Examination [13], which was administered using colloquial/spoken Taiwanese, to indicate no cognitive impairment; and (4) give oral consent to participation.

\section{Procedures}

The study comprised two parts. First, the WHOQOL-BREF (TV) was developed in compliance with the WHOQOL guidelines $[14,15]$. This development process began with determination of four types of Taiwanese scale descriptors $[16,17]$. The WHOQOL-BREF was subsequently translated from English to colloquial Taiwanese. Following this, cognitive debriefing was implemented on another group of 67 elderly Taiwanese in order to verify the conceptual equivalence and appropriateness of the words used. The subjects included 23 females and 44 males who were, on average, 75 years old and met the four selection criteria above. They were recruited from five long-term care institutions throughout four major areas of Taiwan. The cognitive debriefing was carried out by interviewing subjects with the translated WHOQOL-BREF items and then asking for their subjective interpretations of the meaning of each item. After modifying any misleading words, a draft version was translated back into English to examine any possible conceptual discrepancies in equivalence. Several rounds of discussions by a panel of experts led to the final version. The contents were recorded by a female, who enunciated the questions clearly in Taiwanese, using an audio recorder. The choice of a female voice was made at the recommendation of a speech specialist (our team member). The specialist pointed out that, in spoken Taiwanese, which has eight tonal patterns, a female voice is easier than a male voice for the elderly to understand. The recording was made to reduce variability in interviewers' administration of the questionnaire.

The second part of the study was to validate the WHOQOL-BREF (TV). For the validity investigation, the WHOQOL-BREF (TV) was administered face-to-face to each subject by interviewers with assistance of the audio player. Three interviewers received two-hour training by the first author as well as a minimum of three interview practice sessions under supervision. These trained interviewers conducted the WHOQOL-BREF (TV) interviews separately in each assigned institution by following the standardized interview procedure [18] such as subject invitation, audio player installation, and interview administration. Prior to the formal WHOQOL-BREF (TV) interview, each subject was given one practice item to provide familiarity with the audio player-assisted mode of interview. During the formal WHOQOL-BREF (TV) interview, the interviewers played/stopped the audio player when appropriate and recorded the subjects' responses on each item. Replaying of the questions and their scale descriptors was allowed to ensure that the subjects understood the questions and descriptors.

Following the WHOQOL-BREF (TV) interview, a health-related QOL (HRQOL) visual analogue scale (VAS), a self-evaluation health status question, and personal information were collected with the assistance of an audio player. All the interview materials and assessment instruments were translated into colloquial Taiwanese and 
were pre-recorded into the audio player, in order to ensure that the interview was conducted in a standardized manner. The subjects whose QOL remained stable, as determined by additional questions about their self-reported QOL/ health status, were interviewed again two weeks later to determine the test-retest reliability of the WHOQOLBREF (TV).

\section{Instruments}

The WHOQOL-BREF (TV) included 28 items, consisting of 26 standard items from the original WHOQOL-BREF and two culturally relevant items [8, 9]. The 26-item standard WHOQOL-BREF contains two generic items (overall QOL and general health), and the remaining 24 items can be further classified into 4 domains: physical ( 7 items), psychological (6 items), social relationships (3 items), and environment ( 8 items). The two cultural items were "Do you feel respected by others?", which was included in the social relationships domain, and "Are you usually able to get the things you like to eat?" in the environment domain [8, 9].

The HRQOL VAS was used to evaluate a subject's level of satisfaction on his/her overall HRQOL, in which subjects specify the value on a $20 \mathrm{~cm}$ VAS with the left end indicating 0 (worst) and the right end 100 (best). In addition, the self-evaluation health status question required subjects to assess their current health status by selecting 1 of 5 response options: "Very poor", "Poor", "Neither good nor poor", "Good", and "Very good". Both the HRQOL VAS and the self-evaluation health status question were used to examine the convergent validity of the WHOQOL-BREF (TV).

Data analysis

The distributions of domain and item scores as well as the percentage of subjects with missing values for each item were calculated. Cronbach's alpha was used to evaluate the internal consistency of each domain of the WHOQOLBREF (TV). The corrected item-domain correlation was also reported to evaluate the homogeneity of the items in each domain. The intraclass correlation coefficient (ICC) was calculated to determine the test-retest reliability of domain scores of the first and second administrations. The weighted kappa was employed to examine the test-retest reliability of each item of the WHOQOL-BREF (TV).

Four validity indicators were examined. First, the criterion-related validity was studied by measuring the strength of the Pearson $r$ correlation between each item/domain and two criteria, i.e., Q1 (Overall QOL) and Q2 (General health) in this study. Second, convergent validity was determined by examining the relationship between each item/domain of the WHOQOL-BREF (TV) and both the HRQOL VAS and the self-evaluation health status measuring similar constructs, using the Pearson $r$ correlation coefficient. Third, the discriminant validity of the WHOQOL-BREF (TV) was evaluated by performing an analysis of covariance, adjusting for gender, age, and cognitive status, on the subjects' self-evaluation health status. The gender, age, and cognitive status were adjusted because these characteristics might affect the subjective quality of life. Fourth, the confirmatory factor analysis (CFA) was conducted to examine construct validity with the LISREL 8 software [19]. The initial CFA was performed on each domain, using the corresponding items as potential indicators. A second-order factoring was conducted on the four domains with their corresponding indicators as a whole QOL model. The comparative fit index (CFI) was used to determine whether the proposed four factors of the WHOQOL-BREF (TV) were appropriate.

\section{Results}

The study recruited 228 subjects at the first evaluation, 144 of whom were re-assessed after 2 weeks. A total of 84 subjects were lost to follow up largely due to unavailability or disinterest. There were no significant differences in gender, age, and education level between those who completed the second evaluation and those lost to follow-up. The detailed characteristics of the samples are tabulated in Table 1. Table 2 shows descriptive statistics of the items and domains of the WHOQOL-BREF (TV). The floor and ceiling effects (i.e., the percentage of minimum or maximum scores $<10 \%)$ in each domain score were low $(0.4 \%-$ 9.5\%). However, the ceiling effects for Q1 (37.1\%) and Q2 $(26.8 \%)$ were notable, but their floor effects were acceptable $(3.6 \%$ and $6.6 \%$, respectively). The missing values for all items were lower than $10.0 \%$, except for $18.4 \%$ on item 21, which was "How satisfied are you with your sex life?" in the social relationships domain.

\section{Reliability}

The estimated values of Cronbach's alpha for the physical, psychological, social relationships, and environment domains were $0.68,0.70,0.72$, and 0.80 , respectively, indicating acceptable internal consistency. On corrected itemdomain correlation in each domain, all but one individual item had reasonable correlation coefficients (0.21-0.62) which were above the minimum value of 0.2 [20]. Item 4, which asked "How much do you need any medical treatment to function in your daily life?", showed a poor item-domain relationship (-0.03). In addition, the ICC values (0.73-0.79) at the domain level (Table 3) and 
Table 1 Demographic data of subjects that participated in the first and second evaluations

\begin{tabular}{|c|c|c|}
\hline Characters & First evaluation $(n=228)$ & Second evaluation $(n=144)$ \\
\hline \multicolumn{3}{|l|}{ Gender, $n(\%)$} \\
\hline Male & $141(61.8)$ & $87(60.4)$ \\
\hline Female & $87(38.2)$ & $57(39.6)$ \\
\hline \multicolumn{3}{|l|}{ Age (year) } \\
\hline Mean \pm SD & $75.2 \pm 6.5$ & $75.0 \pm 6.9$ \\
\hline Range & $57-101$ & $57-101$ \\
\hline \multicolumn{3}{|l|}{ Education level, $n(\%)$} \\
\hline Illiterate & $88(38.6)$ & $56(38.9)$ \\
\hline Elementary school & $140(61.4)$ & $88(61.1)$ \\
\hline \multicolumn{3}{|l|}{ Marital status ${ }^{\mathrm{a}}, n(\%)$} \\
\hline Single & $107(47.2)$ & $64(44.7)$ \\
\hline Married/Living together & $16(7.0)$ & $8(5.6)$ \\
\hline Divorced/Separated & $22(9.7)$ & $15(10.5)$ \\
\hline Widowed & $82(36.1)$ & $56(39.2)$ \\
\hline \multicolumn{3}{|l|}{ Cognitive status } \\
\hline $\mathrm{MMSE}^{\mathrm{b}}$, Mean $\pm \mathrm{SD}$ & $22.8 \pm 3.7$ & $23.0 \pm 3.5$ \\
\hline \multicolumn{3}{|c|}{ Self-reported health status ${ }^{\mathrm{c}}, n(\%)$} \\
\hline Very poor & $24(10.9)$ & $17(12.2)$ \\
\hline Poor & $33(14.9)$ & $22(15.8)$ \\
\hline Neither good nor poor & $92(41.6)$ & $51(36.7)$ \\
\hline Good & $44(19.9)$ & $28(20.2)$ \\
\hline Very good & $28(12.7)$ & $21(15.1)$ \\
\hline
\end{tabular}

a 1 missing datum at both evaluations

b MMSE: the Mini-Mental State Examination

c 7 and 5 missing data at the $1^{\text {st }}$ and $2^{\text {nd }}$ evaluations, respectively

Table 2 Score distribution of the Taiwanese interview version of the WHOQOL-BREF

\begin{tabular}{lclcc}
\hline Item/domain (possible score range) & Mean $\pm \mathrm{SD}^{\mathrm{a}}$ & Floor effect $(\%)$ & Ceiling effect $(\%)$ & Missing value $(\%)$ \\
\hline Overall QOL, Q1 (1-5) & $3.8 \pm 1.1$ & 3.6 & 37.1 & 1.8 \\
General health, Q2 (1-5) & $3.5 \pm 1.1$ & 6.6 & 26.8 & 0.0 \\
Physical (4-20) & $13.5 \pm 2.9$ & 0.9 & 1.8 & $0.4-7.9$ \\
Psychological (4-20) & $13.3 \pm 3.1$ & 0.4 & 2.2 & $0.0-9.5$ \\
Social relationships (4-20) & $14.3 \pm 3.4$ & 1.4 & 9.5 & $0.0-18.4$ \\
Environment (4-20) & $14.2 \pm 2.8$ & 0.4 & 2.6 & $0.0-6.6$ \\
\hline
\end{tabular}

${ }^{\text {a }}$ Standard deviation

weighted $\kappa$ values $(0.35-0.68)$ at the item level between two evaluations demonstrated acceptable test-retest reliability of domain and item scores.

\section{Validity}

Table 3 shows the results of the criterion-related and convergent validity for the WHOQOL-BREF (TV). All domain scores were fairly to moderately correlated with Q1 $(r \geq 0.32)$, Q2 $(r \geq 0.34)$, the level of satisfaction on HRQOL $(r \geq 0.39)$, and the self-evaluation health status
( $r \geq 0.44)$. At the item level, all but one individual item exhibited weak to fair relationships with the aforementioned indicators $(0.16 \leq r \leq 0.68, p<0.05)$. Only item 4 demonstrated an extremely poor or nonexistent relationship $(-0.07 \leq r \leq-0.01)$. The observations indicated that all but item 4 of the four domains exhibited reasonable criterionrelated and convergent validity.

The four first-order CFA showed that the CFI for physical, psychological, social relationships, and environment domains were $0.95,0.99,1.00$, and 0.95 , respectively, suggesting adequate construct validity. However, item 4 
Table 3 Intraclass correlation coefficient for test-retest reliability and the Person's $r$ coefficient for the criterion-related and convergent validity of the Taiwanese interview version of the WHOQOL-BREF

\begin{tabular}{|c|c|c|c|c|c|}
\hline \multirow[t]{2}{*}{ Domain } & \multirow[t]{2}{*}{ Test-retest reliability } & \multicolumn{2}{|c|}{ Criterion-related validity } & \multicolumn{2}{|c|}{ Convergent validity } \\
\hline & & $\mathrm{Q} 1^{\mathrm{a}}$ & $\mathrm{Q} 2^{\mathrm{b}}$ & HRQOL VAS ${ }^{\mathrm{c}}$ & Self-evaluation health status \\
\hline Physical domain & 0.79 & 0.33 & 0.50 & 0.50 & 0.55 \\
\hline Psychological domain & 0.77 & 0.40 & 0.45 & 0.48 & 0.50 \\
\hline Social relationships domain & 0.73 & 0.32 & 0.34 & 0.39 & 0.44 \\
\hline Environment domain & 0.77 & 0.47 & 0.39 & 0.52 & 0.45 \\
\hline
\end{tabular}

${ }^{a}$ Indicates the overall QOL item

b Indicates the general health item

c Indicates the level of satisfaction on health-related QOL, measured by a $0-100$ visual analogous scale.

* All values achieved statistical significance, i.e., $p<0.05$

did not exhibit a significant factor loading in the physical domain. This item remained a misfit item within the second-order factoring model (Fig. 1); however, the high model-fit index $(\mathrm{CFI}=0.95)$ supported the construct validity of the WHOQOL-BREF (TV), with the exception of item 4. In addition, all four domain scores of the subjects were different in terms of their self-perceived health status (Table 4), supporting the discriminant validity of the WHOQOL-BREF (TV).

\section{Discussion}

To the best of our knowledge, the current study is the first to apply the audio player-assisted interview mode in developing a QOL questionnaire for people using primarily spoken language in their daily lives. This study found that the WHOQOL-BREF (TV), except for the item related to dependence on medication, had acceptable reliability (internal consistency, corrected item-domain correlation, and test-retest reliability) and reasonable validity (criterion-related, convergent, discriminant, and construct validity). Furthermore, most elderly subjects accepted the audio player-assisted mode of interview, according to the interviewers' observations. Approximately a quarter of the subjects in our sample appeared at first to be unfamiliar with this type of interview. Replying to the questions with their own answers immediately after the questions was presented rather than listening to the scale descriptors and choosing one. These subjects, however, could complete the entire interview in the proper way after receiving further instruction and practice. With the aforementioned positive feedback and the acceptable psychometric findings, this study supports the use of an audio player-assisted interview, which can provide a practical method of assessing QOL for people who use only spoken language.

The WHOQOL-BREF (TV) was found to possess psychometric properties similar to those of the WHOQOL-
BREF Mandarin Chinese version [8, 9] and the English version [2]. However, the WHOQOL-BREF (TV) contained some specific items that call for careful attention. Item 4 (medication) was the only item which showed neither reasonable corrected item-domain correlation nor acceptable criterion-related and convergent validity. The high level of medication use in the elderly population seems necessary to support their function in daily life [21]. It could be that the requirement of medication becomes a daily routine for the elderly, thus making this item less important to their physical health and/or irrelative to other heath-related variables. The physical domain showed improved internal consistency (data not shown) after the deletion of item 4. These observations imply that item 4 in the WHOQOL-BREF (TV) may be deleted or needs to be revised. Moreover, the two generic items in the WHOQOLBREF (TV) showed notable ceiling effects and therefore the ceiling effects might limit their abilities to discriminate people with high QOL. However, the discriminant validity of these two generic items was not significantly damaged according to the study results. In addition, item 21 (sexual life) in the WHOQOL-BREF (TV) exhibited a high missing value for Taiwanese-speaking elderly. The high missing response rate on item 21 was consistent with the findings of the WHOQOL-BREF Mandarin Chinese version [22, 23] and might be due to a substantial proportion of elderly people living alone (e.g., $92 \%$ in this study). It could also be that Chinese/Taiwanese culture discourages explicit self expression of such sexual desires [22, 23]. Hwang et al. [23] and Power et al. [21] have suggested that considering intimacy other than sexual intercourse might increase the response from the elderly on this item. Fortunately in this study, this flaw in item 21 did not compromize the reliability and validity of the social relationship domain.

It is noted that, except for item 4, the WHOQOL-BREF (TV) items associated weakly or fairly with the chosen criteria, such as overall QOL or health status. The weak criterion-related and convergent validity at the item level 
Fig. 1 Confirmatory factor analyses on the second-order factor structure $(N=228)$.

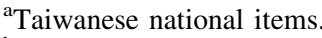

${ }^{b}$ Indicates non-normed fit index.

${ }^{\mathrm{c}}$ Indicates comparative fit index

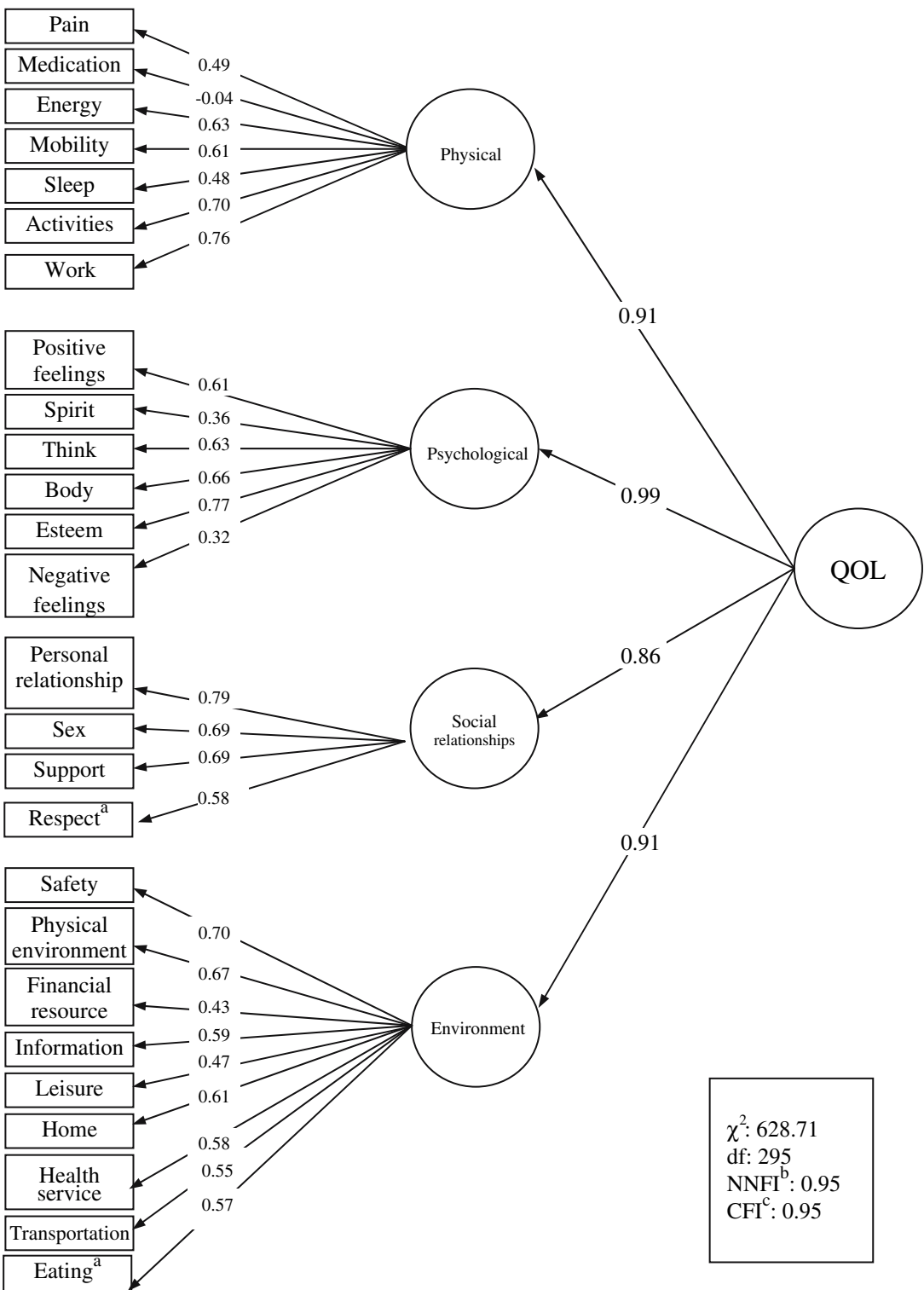

Table 4 Discriminant validity of the Taiwanese interview version of the WHOQOL-BREF

\begin{tabular}{|c|c|c|c|c|c|c|c|}
\hline \multirow[t]{2}{*}{ Item/Domain } & \multicolumn{5}{|c|}{ Self-evaluation health status ${ }^{\mathrm{a}}$ (mean) } & \multirow[t]{2}{*}{$F$-statistics ${ }^{\mathrm{b}}$} & \multirow[t]{2}{*}{$p$-value } \\
\hline & Very poor $(N=24)$ & Poor $(N=33)$ & $\begin{array}{l}\text { Neither good nor } \\
\text { poor }(N=92)\end{array}$ & Good $(N=44)$ & Very good $(N=28)$ & & \\
\hline Overall QOL (Q1) & 3.2 & 3.4 & 3.7 & 4.3 & 4.5 & 5.6 & $<0.01$ \\
\hline General health (Q2) & 2.6 & 3.2 & 3.3 & 4.2 & 4.5 & 12.3 & $<0.01$ \\
\hline Physical domain & 10.8 & 12.2 & 13.1 & 15.2 & 16.1 & 13.7 & $<0.01$ \\
\hline Psychological domain & 10.0 & 12.4 & 12.9 & 15.3 & 15.3 & 14.1 & $<0.01$ \\
\hline Social domain & 12.4 & 13.0 & 13.6 & 15.3 & 17.4 & 9.1 & $<0.01$ \\
\hline Environment domain & 12.6 & 13.5 & 13.4 & 15.4 & 16.9 & 11.9 & $<0.01$ \\
\hline
\end{tabular}

${ }^{a} N=221,7$ missing data

b An analysis of covariance adjusting gender, age, and cognitive status 
were also found in previous studies $[9,24]$, possibly because the subjects' overall QOL or health status involve multiple factors (e.g., physical, psychological, and environmental factors), whereas each individual item relates to a certain narrow QOL facet. In addition, this study had a substantial sample size for the CFA operation (more than 200 subjects) according to suggestions from previous studies [25, 26]. However, CFA results showed low or even negative factor loadings for item 4 (medication) in the physical domain, as well as for item 6 (spirit) and item 26 (negative feelings) in the psychological domain. These results indicated that these items, especially item 4 , might not measure the underlying construct inherent in their corresponding domains and thus might require modification or removal.

In conclusion, this study suggests that the newly developed audio player-assisted interview version of the WHOQOL-BREF (TV) provides a reliable and valid QOL instrument for Taiwanese-speaking elderly people. Future studies that examine agreement between the WHOQOLBREF (TV) and the Mandarin Chinese version of WHOQOL-BREF are warranted to determine whether the results obtained from the two versions can be regarded as identical for individual/group comparisons.

Acknowledgements This study was supported by research grants from the National Health Research Institute (NHRI-EX95-9504PP and NHRI- EX95-9512PI). The authors would like to thank Ms Vanessa Carson for her assistance in editing this manuscript prior to submission.

\section{References}

1. Skevington, S. M., Sartorius, N., \& Amir, M. (2004). Developing methods for assessing quality of life in different cultural settings. The history of the WHOQOL instruments. Social Psychiatry and Psychiatric Epidemiology, 39(1), 1-8.

2. The WHOQOL Group. (1998). Development of the World Health Organization WHOQOL-BREF quality of life assessment. Psychological Medicine, 28(3), 551-558.

3. Leung, K. F., Wong W. W., Tay, M. S., Chu, M. M., \& Ng S. S. (2005). Development and validation of the interview version of the Hong Kong Chinese WHOQOL-BREF. Quality of Life Research, 14(5), 1413-1419.

4. Jang, Y., Hsieh, C. L., Wang, Y. H., \& Wu, Y. H. (2004). A validity study of the WHOQOL-BREF assessment in persons with traumatic spinal cord injury. Archives of Physical Medicine and Rehabilitation, 85(11), 1890-1895.

5. Fang, C. T., Hsiung, P. C., Yu, C. F., Chen, M. Y., \& Wang, J. D. (2002). Validation of the World Health Organization quality of life instrument in patients with HIV infection. Quality of Life Research, 11(8), 753-762.

6. Izutsu, T., Tsutsumi, A., Islam, A., et al. (2005). Validity and reliability of the Bangla version of WHOQOL-BREF on an adolescent population in Bangladesh. Quality of Life Research, 14(7), 1783-1789.
7. Wang, W. C., Yao, G., Tsai, Y. J., Wang, J. D., \& Hsieh, C. L. (2006). Validating, improving reliability, and estimating correlation of the four subscales in the WHOQOL-BREF using multidimensional Rasch analysis. Quality of Life Research, 15(4), $607-620$

8. Yao, G., Chung, C. W., Yu, C. F., \& Wang, J. D. (2002). Development and verification of validity and reliability of the WHOQOL-BREF Taiwan version. Journal of the Formosan Medical Association, 101(5), 342-351.

9. The WHOQOL-Taiwan Group. (2001). The user's manual of the development of the WHOQOL-BREF Taiwan Version. 1st rev. ed. Taipei: Taiwan WHOQOL Group.

10. Department of Health (R.O.C). (2000). Vital statistics in 1999. Taipei: Department of Health, Executive Yuan, Taiwan, Republic of China.

11. Tsai, S. (2001). Language usage and occupational stratification in Taiwan: Comparing ethnic differences among men. Taiwanese Sociology (Taipei), 1, 65-111.

12. Higginson, I. J., \& Carr, A. J. (2001). Measuring quality of life: Using quality of life measures in the clinical setting. British Medical Journal, 322(7297), 1297-1300.

13. Folstein, M. F., Folstein, S. E., \& McHugh, P. R. (1975). "Minimental state". A practical method for grading the cognitive state of patients for the clinician. Journal of Psychiatric Research, 12(3), 189-198.

14. World Health Organization. (1993). WHOQOL Study Protocol. WHO/MNH/PSF/93.9, Geneva: WHO.

15. World Health Organization. (1994). WHOQOL Protocol for New Centers. WHO/MNH/PSF/94.4, Geneva: WHO.

16. Chien, C. W., Wang, J. D., \& Hsieh, C. L. (2004). Determining scale descriptors of the interview version of WHOQOL for Minnan people. Quality of Life Research, 13(9), 1529.

17. Chien, C. W., Wang, J. D., Yao, K. P., Li, C. A., \& Hsieh, C. L. (2005). Minnan version of determining scale descriptors of the interview questionnaire of WHOQOL. Formosan Journal of Medicine (Taipei), 9, 584-594.

18. The WHOQOL-Taiwanese Group. (2007). Development and instruction of the WHOQOL-BREF Taiwanese Interview Version. Taipei: Taiwanese WHOQOL Group.

19. Joreskog, K. G., \& Sorbom D. (1993). LISREL 8: Structural equation modeling with the SIMPLIS command language. Hillsdale, NJ: Erbaum Assoicates.

20. Streiner, D. L., \& Norman, G. L. (1995). Health Measurement Scale: A practical guide to their development and use, (2nd ed.). Oxford: Oxford University Press.

21. Power, M., Quinn, K., \& Schmidt, S. (2005). Development of the WHOQOL-old module. Quality of Life Research, 14(10), 2197-2214.

22. Lai, K. L., Tzeng, R. J., Wang, B. L., Lee, H. S., Amidon, R. L., \& Kao, S. (2005). Health-related quality of life and health utility for the institutional elderly in Taiwan. Quality of Life Research, 14(4), 1169-1180.

23. Hwang, H. F., Liang, W. M., Chiu, Y. N., \& Lin, M. R. (2003). Suitability of the WHOQOL-BREF for community-dwelling older people in Taiwan. Age Ageing, 32(6), 593-600.

24. Jaracz, K., Kalfoss, M., Gorna, K., \& Baczyk, G. (2006). Quality of life in Polish respondents: psychometric properties of the Polish WHOQOL-Bref. Scandinavian Journal of Caring Sciences, 20(3), 251-260.

25. Crowley, S. L., \& Fan, X. (1997). Structural equation modeling: Basic concepts and applications in personality assessment research. Journal of Personality Assessment, 68(3), 508-531.

26. Boomsma, A. (1987). The robustness of maximum likelihood estimation in structural equation models. In P. Cuttance \& R. Ecob (Eds.), Structural equation modeling by example, (pp. 160-188). New York: Cambridge University Press. 